\title{
Regular Expression Patterns
}

\author{
Niklas Broberg \\ d00nibro@dtek.chalmers.se
}

\author{
Andreas Farre \\ d00farre@dtek.chalmers.se
}

Chalmers University of Technology

\author{
Josef Svenningsson \\ josefs@cs.chalmers.se
}

\begin{abstract}
We extend Haskell with regular expression patterns. Regular expression patterns provide means for matching and extracting data which goes well beyond ordinary pattern matching as found in Haskell. It has proven useful for string manipulation and for processing structured data such as XML. Regular expression patterns can be used with arbitrary lists, and work seamlessly together with ordinary pattern matching in Haskell.
\end{abstract}

Our extension is lightweight, it is little more than syntactic sugar. We present a semantics and a type system, and show how to implement it as a preprocessor to Haskell.

\section{Categories and Subject Descriptors}

D.3.2 [Language Classifications]: Applicative (functional) languages; D.3.3 [Language Constructs and Features]: Patterns

\section{General Terms}

Languages

\section{Keywords}

Regular expressions, pattern matching, Haskell

\section{Introduction}

Pattern matching as found in many functional languages is a nice feature. It allows for clear and succint definitions of functions by cases and works very naturally together with algebraic data types. But sometimes ordinary pattern matching is not enough. A distinct feature of this form of pattern matching is that it only examines the outermost constructors of a data type. While this allows for efficient implementations it is also a rather limited construct for analysing and retrieving data.
Permission to make digital or hard copies of all or part of this work for personal or classroom use is granted without fee provided that copies are not made or distributed for profit or commercial advantage and that copies bear this notice and the full citation on the first page. To copy otherwise, to republish, to post on servers or to redistribute to lists, requires prior specific permission and/or a fee.

ICFP'04, September 19-21, 2004, Snowbird, Utah, USA

Copyright 2004 ACM 1-58113-905-5/04/0009 ...\$5.00
A well-known example of a construct that provides deeper and more complex retrievals are regular expressions for strings. While this is not a very common feature among programming languages it is one of the key constructs that have made Perl so popular. Regular expressions are ideal for various forms of string manipulation, text extraction etc, however, they remain a very domain specific and adhoc construct targeted only for one particular data structure, namely strings.

On another axis we find the recent trend in XML centric languages. The first attempts at such languages used the ordinary pattern matching facility of functional languages to analyze XML fragments [MS99]. This was found to be too restrictive, so in order to be able to express more sophisticated patterns and transformations on XML fragments the notion of regular expression patterns were invented. Examples of languages including this feature are XDuce [HP03] and CDuce [BCF03]. While this is a great boost for the XML programmer, in the case of XDuce it only works for XML data and not for any other data. Furthermore those pattern matching constructs are closely tied to rather sophisticated type systems which makes them somewhat heavyweight.

In this paper we extend Haskell with regular expression patterns. Our extension has the following advantages:

- Our proposal is lightweight. It is hardly more than syntactic sugar. Most notably it does not require any complex additions to the type system.

- It works for arbitrary lists. It is a general construct and not tied to a specific data type for elements. But it should be noted that it works in particular for strings since strings are just lists of characters in Haskell.

- It fits seamlessly with the ordinary pattern matching facility found in Haskell.

In this paper we give a detailed semantics and type system of regular expression patterns. The extension has been implemented as a preprocessor to Haskell, and we sketch the implementation

While we have chosen to focus on Haskell in this paper there are very little Haskell specific details. We are quite confident that our proposal could be adapted to any similar functional language.

In recent years a number of papers have been devoted to developing efficient pattern matching and efficient regular matching [Fri04, HM03, Lev03]. This is not the concern of this paper. Although efficiency is an important consideration we focus only on language design. 
Another issue that we do not address is the question of overlapping and exhaustive patterns. We are confident that the existing techniques developed for XML centric languages will do the job nicely [HVP00]. Note also that in general it is undecidable to check whether patterns are overlapping or non-exhaustive in Haskell because of guards, so in our setting it is something of a non-issue.

\section{Regular expression patterns by example}

\subsection{Ordinary pattern matching}

Assume that we have the following datatype representing an entry in an address book.

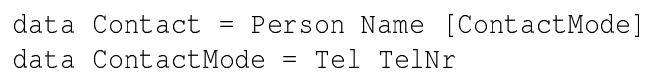

We can assume that the types Name and TelNr are type synonyms for String. The reason for not inlining TelNr in contact is because we will later want to add other means of contact, e.g. email addresses, to our address book.

Now consider two different functions that extract information from a contact; firstTel will return the first $\mathrm{TelNr}$ in the list of contact modes associated with a contact. lastTel will analogously return the last associated TelNr. The first is easy to write using simple pattern matching on a contact:

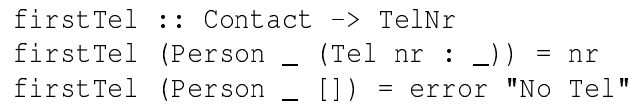

The second function, although its functionality is very similar to firstTel, cannot be written in the same simple way. We must instead resort to recursion and an auxiliary function to step through the list until we reach the end.

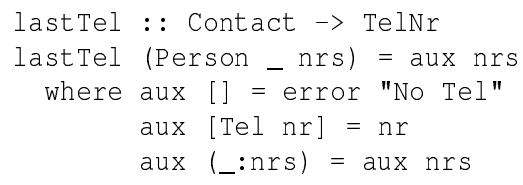

Although the two functions have very similar functionality, only one of them can be written using direct pattern matching. Why is this so? The answer lies, of course, in the list datatype. A (nonempty) list has a head and a tail, so extracting the first element is easy. To get to the last element however, we must recursively look at the tail for its last element. In other words, we must first match on the structure of the list, before being able to look at the elements.

Haskell has a construct for matching directly on the elements of a list, but only for fixed-size lists. If we know that a contact never has more than three phone numbers, we could write lastTel as (we will ignore the erroneous case from now on)

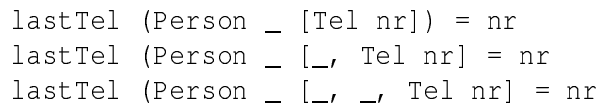

Clearly this is not a very good solution. Even for this very small task we must write far more than we are comfortable with, and for larger lists or more complex datatypes this approach quickly becomes infeasible. What we need is a way of saying "match a list containing a $\mathrm{Tel}$, preceded by any number of other elements". This is where regular expression patterns enter the picture.

\subsection{Regular expression patterns}

Mathematically a regular expression defines a regular language, where language in this context means a (possibly infinite) set of words, and each word is a sequence of elements taken from some alphabet. We can use a regular expression as a validator and try to match an arbitrary word against it to find out if the word belongs to that regular language or not. The basic regular expression operators are repetition, concatenation and choice. Concatenation is straightforward, $a b$ means $a$ followed by $b$. Choice $(a \mid b)$ means either $a$ or $b$. Repetition $a *$ means zero or more occurrences of $a$. Repetition can be defined using choice and recursion as $a *=\varepsilon \mid a a *$ where $\varepsilon$ denotes the empty sequence. As an example, consider the regular expression $e=a * \mid b *$. The language defined by $e$, denoted $L(e)$, is the set of all words consisting of only $a$ 's or only $b$ 's, including the empty word. We have that $a a \in L(e), b b b \in L(e)$, but $a b \notin L(e)$. In other words, $a a$ and $b b b$ both match the regular expression $e$, but $a b$ doesn't.

This notion of treating a regular expression as a validator is very similar to the concept of pattern matching in Haskell. We take a Haskell value (a word) and a pattern (a regular expression) and try to match them, getting a yes or no as the result. Combining these two concepts is straight-forward, yielding what we call regular expression patterns. As noted, a regular expression can be matched against a sequence of elements from some alphabet. Lifting this idea into Haskell, a regular expression pattern can be matched against a list of elements of some datatype. When we speak of a sequence, we mean a sequence of elements in the abstract sense. In contrast, when we speak of a list, we mean the list datatype that is used to encode sequences in Haskell.

Returning to our lastTel function, we can now easily write it with a single pattern match by using a repetition regular expression pattern:

lastTel (Person _ [_*, Tel nr]) $=$ nr

We write concatenation using commas as with ordinary Haskell lists, and we denote repetition with *. As we can see from the example, regular expression patterns are actually more flexible than bare regular expressions. A regular expression is built from elements of some alphabet, the same alphabet that the words it may match are built from. A regular expression pattern on the other hand is built from patterns over elements of some datatype, allowing us to use constructs like wildcards and pattern variables. We use the term regular expression pattern both for the subpatterns (repetition, choice etc) and for a top-level list pattern that contains the former. It should be clear from the context which we are referring to.

\subsection{Repetition and Ambiguities}

Let us see what else we can do with regular expression patterns. First, as promised, we extend our datatype with email addresses.

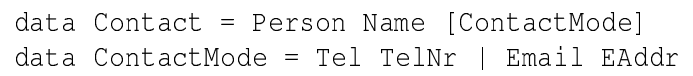

If we only have ordinary pattern matching we cannot even write first Tel without resorting to recursion and auxiliary functions.

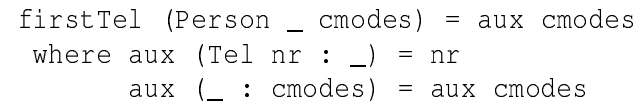

Using a regular expression pattern, we can write it in one go: 
firstTel (Person _ [(Email_ _), Tel nr, _*]) = nr

The straight-forward intuition of the pattern above is that the first Tel in the list is preceded by zero or more Emails (but no Tels), and any number of other elements may follow it. We can easily write last $T e l$ in a similar way as

lastTel (Person_ [_*, Tel nr, (Email__* ]) = nr

But seeing these two definitions leads to an interesting question: What happens if we write the function

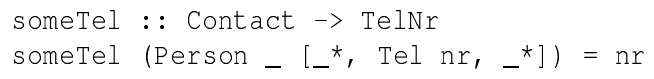

i.e. where the Tel in question may both be preceded and succeeded by other Tels? Clearly this pattern is ambiguous, since if we match it to e.g. Person "Niklas" [Tel 12345, Tel 23456, $\mathrm{Tel}$ 34567] we can derive a match for either of the three TelNrs to be bound to $n$ r, by letting the first _* match either 0,1 or $2 \mathrm{Tels}$. To disambiguate such issues, we adopt the policy that a repetition pattern will always match as few elements as possible while still letting the whole pattern match the given list. In standard terminology, our repetition regular patterns are non-greedy. This policy means that someTel above will be exactly the same as our firstTel function, since the first _* will now try to match as few elements as possible.

In some cases though, such as lastTel, we want the greedy behavior. To this end we let the programmer specify if a repetition pattern should be greedy by adding an exclamation mark (!) to it, e.g. in the following definition of last Tel:

lastTel (Person [_*!, Tel nr, _*]) = nr

\subsection{Choice patterns}

Now that we've seen the power of repetition patterns, we turn our attention to choice patterns. Assume that we want a function all Tels that returns a list of all telephone numbers associated with a contact. Without regular expression patterns we must once more resort to recursion and auxiliary functions.

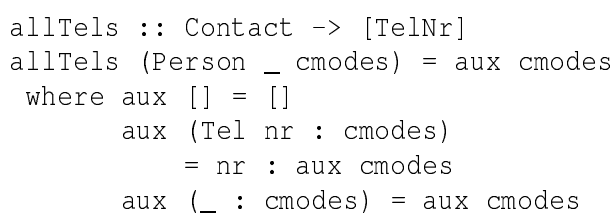

Using a combination of repetition and choice, we can write it as

$$
\text { allTels (Person_ } \left.\left[\left(\text { Tel nr }\left.\right|_{-}\right) *\right]\right)=n r
$$

The intuition here is that each element in the list of contact modes is either a $\mathrm{Tel}$ or something else (_). Every time that we encounter a Tel, we should include the associated TelNr in the result. As the example shows we can achieve this accumulation of TelNrs with a single pattern variable. Since the intuition of a repetition pattern is that its subpattern, i.e. the pattern it encloses, should be matched zero or more times, the same must be true for any pattern variables inside such a pattern. For each repetition, such a variable will match a new value. Clearly the only sensible thing to do is to let that variable bind to a list of all those matched values.

This treatment of variables breaks one aspect of Haskell's linearity property - that the occurrence of a variable in a pattern will bind that variable to exactly one value of the type that it matches. We will therefore call such a variable non-linear. A non-linear variable will be bound to a list of values that it matches, in the order that they were matched (i.e. the order in which they appeared in the matched list). When we speak of a non-linear binding, we mean a binding of a non-linear variable to a list of values. We will also use the terms non-linear context to mean a context in which linear variables cannot appear, and non-linear patterns, by which we mean patterns whose subpatterns will always be matched in a non-linear context.

By the example above we see that a repetition pattern is a nonlinear pattern, and consequently that the variable $n r$ appears in a non-linear context. Similarly a choice pattern is also non-linear. If we remove the repetition from the regular expression pattern in allTels we get the pattern [Tel $\mathrm{nr} \mathrm{I}_{\mathbf{2}}$ ] for matching a list of exactly one element. If that element is a $\mathrm{Tel}$ we will have a value to bind to nr, but if it is an Email we have none! Thus we still cannot guarantee that a variable gets one value; in this case $n r$ will be bound to a list with zero or one element.

The function all Tels shows how regular expression patterns can be used for filtering a list based on pattern matching. We can go one step further and do partitioning, e.g.

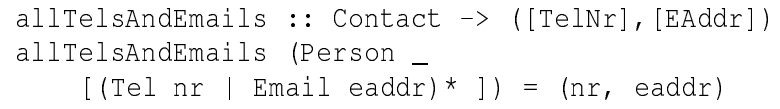

A choice pattern can also be ambiguous if any of its subpatterns overlap, as in

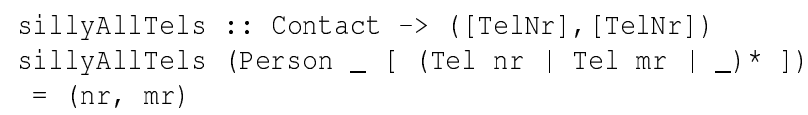

To disambiguate this we adopt a first-match policy, much like that of Haskell pattern matching. Thus we first check if the first subpattern matches, and consider the $k$ :th subpattern only if no pattern $i<k$ matches. Note that we allow choice patterns to contain more than two subpatterns. Choice patterns are right associative so for example [ (Tel $\left.n r|\mathrm{Tel} \mathrm{mr}|_{-}\right)^{*}$ ] is parenthesised like [ (Tel nr | (Tel mr $\left.\left.\left.\mid{ }_{-}\right)\right)^{*}\right]$. Another interesting thing about choice patterns is that we allow a variable to appear in both subpatterns assuming that it binds to values of the same type. For instance, if our datatype for modes of contact was defined as

data ContactMode $=$ Home TelNr $\mid$ Work TelNr

we could define allTels as

allTels (Person _ [(Home nr | Work $n r) *])=n r$

Variables in choice patterns are still non-linear even if they appear in all subpatterns, so the function

singleTel (Person_ [(Home nr | Work nr)]) = nr

will have the type Contact $\rightarrow[\mathrm{TelNr}]$.

\subsection{Subsequences and option patterns}

Regular expressions allow grouping of elements and subexpressions using parentheses. For example, the regular expression $e=$ $(b a) *$ will match the words $b a, b a b a$ etc. To add this feature to our regular expression patterns we need to introduce some new syntax, since using ordinary parentheses in Haskell will denote tuples, as in 
wrongEveryother $[(,, b) *]=b$

We (somewhat arbitrarily) choose to denote subsequences with (/ and /), so a correct function that picks out every other element from a list can be written as

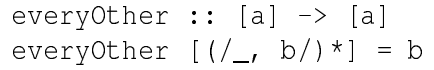

There's a problem with the above definition though; it works for lists of even length only. Surely we want everyother to work for any list. To achieve this we could add another declaration to the one above like

$$
\text { everyother }[(/,, \mathrm{b} /) *, \ldots]=\mathrm{b}
$$

to catch the cases where the list is of odd length too. But couldn't we write these two cases as a single pattern? Indeed we can, using a choice pattern

$$
\text { everyother }[(/,, \mathrm{b} /) *,((/ /) / \ldots)]
$$

where (/ / ) denotes the empty subsequence, $\varepsilon$. However, this pattern is so common that regular expressions define a separate operator, ?, to denote optional regular expressions. The definition of ? is $e ?=e \mid \varepsilon$, and by lifting this to regular expression patterns we can write everyother more compactly as

$$
\text { everyother }[(/,, \mathrm{b} /) *, \ldots ?]=\mathrm{b}
$$

Obviously, optional patterns are non-linear since they can be defined in terms of choice patterns which are non-linear. Just as for a repetition pattern, an optional pattern is non-greedy by default. We also define greedy optional patterns by ?! in analogy with greedy repetition patterns.

\subsection{Non-empty repetition patterns}

There is one more operator to discuss, namely + that is used to denote non-empty repetition. For instance we might require all contacts to have at least one mode of contact registered, either a telephone number or an email, otherwise it is an error. To enforce this we may want to define allTelsAndEmails from above as

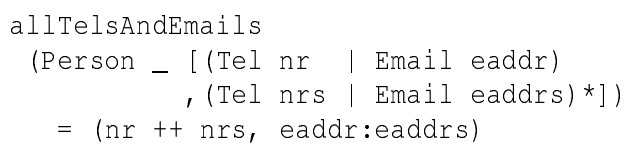

Using + we can define this more compactly as

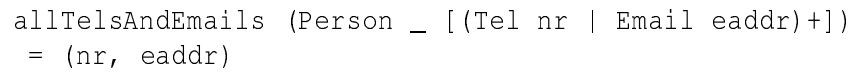

Modulo variables bound, $p+\equiv p p *$. It is non-linear and nongreedy just like *, and there is a greedy counterpart + !.

\subsection{Variable bindings and their types}

Since we can use any Haskell pattern inside regular expression patterns, we can in particular use pattern variables to extract values from the list that we match against, as we have seen in various examples already. Haskell also defines a way to explicitly bind values to a variable using the e operator. E.g. in the declaration

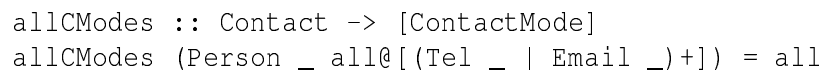

the variable all will be bound to the (non-empty) list of ContactModes associated with a contact. This is a very useful feature to have for regular expression patterns as well, for instance we may want to write a function that picks the first two elements from a list as

$$
\begin{aligned}
& \text { twoFirst }::[a]->[a] \\
& \text { twoFirst }\left[a\left([/ /, \ldots /),{ }^{\star}\right]=a\right.
\end{aligned}
$$

However, adding this feature raises some interesting questions. Firstly, what will the type of a variable bound to a regular expression pattern be? For a subsequence it seems fairly obvious that it will have a list type, but what about repetitions, choices and optional patterns? To this issue there is no obvious right answer, one way might be to let a variable be bound to all elements matched by the subpattern in analogy with implicitly bound variables. We have chosen a slightly different approach in which we assign different types to patterns to mirror the intuition behind them.

Subsequences and repetition patterns will both have list types since they represent sequences. There's a difference between them though; a subsequence is just what the name implies, a subsection of the original sequence. Thus a variable bound to it will always have the same type as the input list, i.e. a list of elements. A repetition pattern on the other hand is a repetition of some subpattern, and so it will have the type of a list of that subpattern. For choice patterns we make use of Haskell's built-in Either type defined as

data Either $\mathrm{a} b=$ Left $\mathrm{a} \mid$ Right $\mathrm{b}$

By using this type we can allow the left and right subpatterns of a choice pattern to have different types, for instance

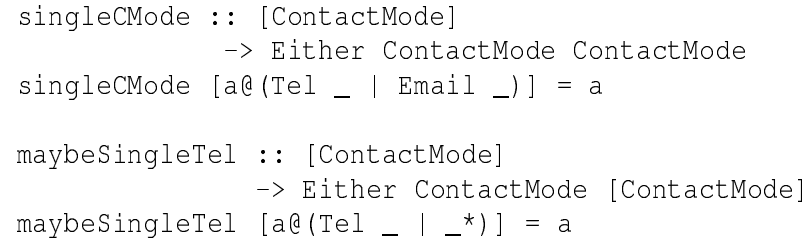

Similarly for optional patterns we use another built-in Haskell type: data Maybe $\mathrm{a}=$ Nothing $\mid$ Just $\mathrm{a}$

so if we write a function

singleorNoTel [(Email__) , a@ (Tel__) ?, (Email_ _)*] =a it will have the type [ContactMode] -> Maybe ContactMode.

One way to think about this is to see the regular expression pattern operators as special data constructors. In an analogy with ordinary Haskell, we don't expect a to have the same type in the two uses a@ (Just _) and (Just a@_). Nor do we expect the a in $a @$ (__) to have the same type as the $a$ in (a@_) ?.

The second issue concerns linear vs. non-linear binding. We have already seen that implicit bindings, i.e bindings that arise from the use of ordinary pattern variables, are context dependent; in linear context they get the ordinary types, whereas in non-linear context they get list types. This context dependence unfortunately makes it easy for the programmer to make mistakes, since it isn't clear just by looking at a variable in the pattern what type it will have. We cannot do anything about implicit bindings, but we can avoid the same problem for explicit binding. Therefore we let the ordinary e operator signify linear explicit binding, the only kind available 
in ordinary Haskell. For non-linear explicit binding we introduce a new operator $\&$ : (read "as cons" or "accumulating as"). The former may not appear in non-linear context, whereas the latter may appear anywhere inside a regular expression pattern. Their differences are shown by the following examples:

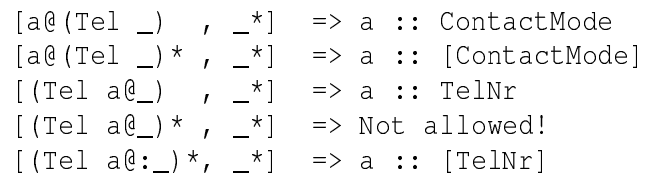

We can define the semantics of implicit bindings in terms of explicit bindings. In linear context we have that a pattern variable a is equivalent to the pattern $a e_{\text {. }}$. This can be seen in the example $\left[\left(\begin{array}{ll}\text { Tel } & a\end{array}\right),-^{\star}\right]$ which is clearly equivalent to [ ( Tel $\left.\left.a e_{-}\right),-^{\star}\right]$. In non-linear context, $\mathrm{a}$ is equivalent to $\mathrm{a}^{\mathrm{e}} \mathrm{:}_{-}$, , as in the examples $\left[\left(\begin{array}{ll}\text { Tel } & a\end{array}\right)^{*},-^{\star}\right]$ and $\left[\left(\operatorname{Tel} a:_{-}\right)^{*},-^{\star}\right]$.

\subsection{Further examples}

Now that we've seen all the basic building blocks that our regular expression patterns consist of, let us put them to some real use.

Traditionally regular expressions have been used in programming languages for text matching purposes, and certainly our regular expression patterns are well suited for this task. As an example, assume we have a specification of a simple options file. An option has a name and a value, written on a single row, where name and value are separated with a colon and a whitespace. Different options are written on different lines. Here are the contents of a sample options file:

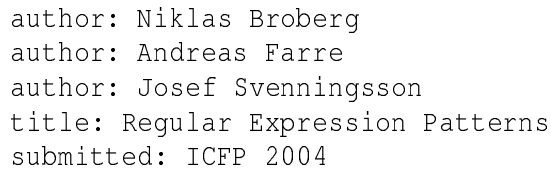

A simple parser for such option files can be written using a regular expression pattern as

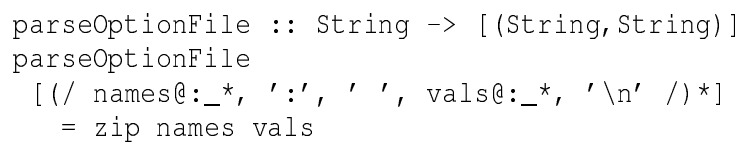

where zip is a function that takes two lists and groups the elements pair-wise.

XML processing is another area that greatly benefits from regular expressions, since "proper pattern matching on XML fragments requires ... matching of regular expressions" [MvV01]. Indeed several recent XML-centric languages (XDuce, CDuce) include regular expressions as part of their pattern matching facilities.

As an example we encode XML in Haskell using a simple datatype

$$
\begin{aligned}
\text { data XML } & =\text { Tag String }[\mathrm{XML}] \\
& \mid \text { PCDATA String }
\end{aligned}
$$

An XML fragment is either a Tag, e.g. $\langle\mathrm{P}\rangle \ldots \quad\langle/ \mathrm{P}\rangle$, which has a name (a String) and a list of XML children, or it is PCDATA (XML lingo for a string inside tags). This model is of course extremely simplified, we've left out anything that will not directly add anything to our example, most notably XML attributes. Now assume that we have a simple XML email format, where a sample email message in this format might look like:

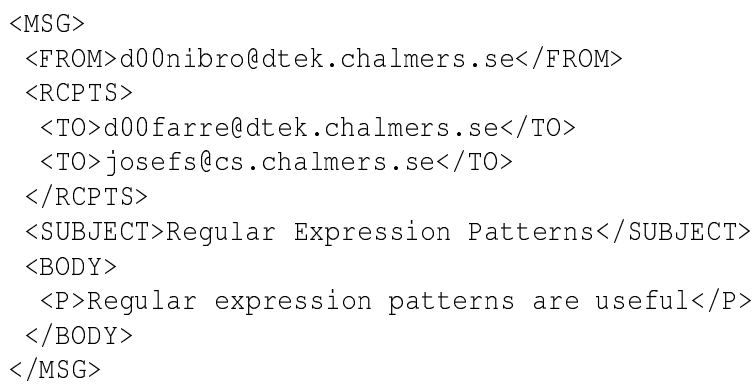

which would be encoded in our XML datatype as

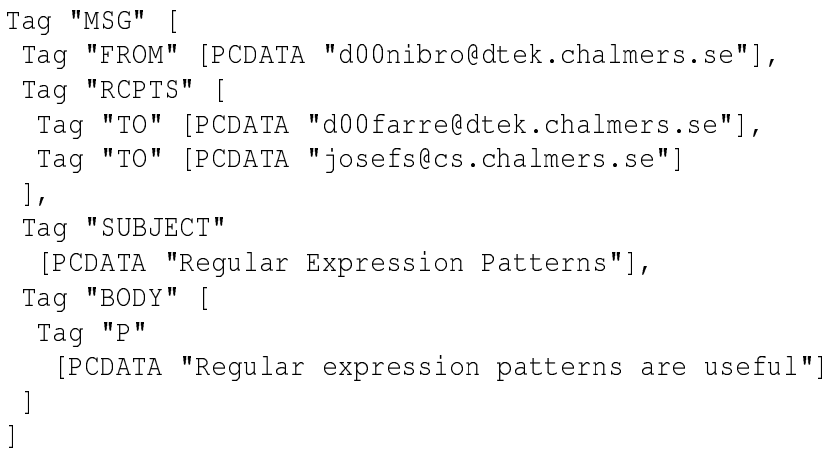

We can write a function to convert messages from this XML format into the standard RFC822 format using regular expression patterns:

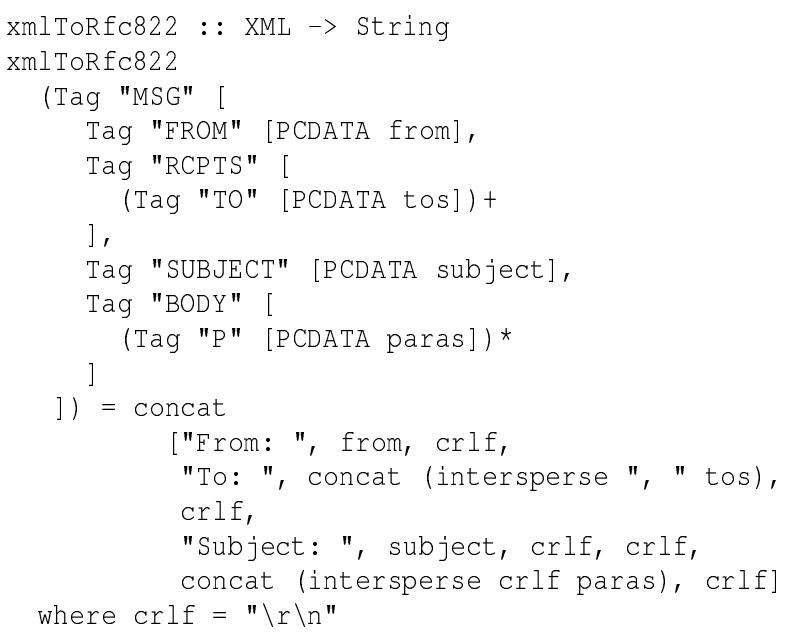

\section{Syntax}

The previous section has gone over all of regular expression patterns by example. This section starts the formal treatment by giving a grammar for the syntax, which can be seen in figure 1 . We refer to the nonterminal for Haskell's ordinary patterns as pattern and extend it with a new production for regular expression patterns.

The concrete syntax is quite close to that of e.g. Perl [Perl] or CDuce [BCF03] with the notable exception that we have nongreedy patterns as default. An extra exclamation mark indicates greediness. 


$$
\begin{aligned}
& \text { pattern } \rightarrow \ldots \\
& \text { regpat } \rightarrow \text { pattern } \\
& \text { regpat '*'['!'] } \\
& \text { regpat '+'['!'] } \\
& \text { regpat '?'['!'] } \\
& \text { regpat "| regpat } \\
& \text { '(/' regpat } \left.{ }_{1} \text {... regpat } \text { reg }^{\prime}\right) \text { ' } \\
& \text { '(' regpat ')' } \\
& \text { var'@'regpat } \\
& \text { var'@:' regpat }
\end{aligned}
$$

Figure 1. Regular expression pattern syntax

Ordinary Haskell patterns are regular expressions patterns. The operators are repetition $(*)$, non-empty repetition $(+)$ and option (?). Furthermore there are choice patterns indicated by a vertical bar and subsequences are enclosed in subsequence brackets. Regular expression patterns can be enclosed in parenthesis. The last two productions are for linear and non-linear variable bindings. Precendence of the operators is as follows: *, +, ?, *!, +! and ?! binds strongest. They are followed by choice patterns which are also right associative. Lastly we have @ and @: which bind weakest. All constructs in regular expression patterns bind stronger than constructor application.

\section{Semantics}

In this section we turn to the formal semantics for regular expression patterns. Our semantics divides natually into two parts; one for linear and one for non-linear patterns. The reason for this division is that variable bindings are treated differently.

\subsection{Structure of semantics}

We give the semantics as an all-match semantics. This leads to possibly ambiguous matches, the same list can be matched in many different ways. Since this may affect how variables are bound to their values we need to disambiguate our rules. We follow the approach taken by Hosoya and Pierce [HP03] and introduce an ordering on the rules indicating which rule will have precedence when several rules can match. The order is given by numbers in the name of the rules, where lower numbers have higher precedence. Intuitively this means that when building the derivation tree for a match, one must always try to use the rule with the highest precedence first, and choose the other rule only if choosing the first rule cannot lead to a match.

Before we begin with the semantics we will define some concepts which will be used in our explanation of the semantics. We will use sets of variable bindings to map variables to values. A variable binding is denoted $x \mapsto v$. In repetition patterns we will need to merge sets of variable bindings with overlapping domains. We use $\uplus$ to this end and define it as follows:

$$
\left\{x_{1} \mapsto v_{1}, \ldots, x_{n} \mapsto v_{n}\right\} \uplus\left\{x_{1} \mapsto v s_{1}, \ldots, x_{n} \mapsto v s_{n}\right\}=
$$$$
\left\{x_{1} \mapsto v_{1}+v s_{1}, \ldots, x_{n} \mapsto v_{n}+v s_{n}\right\}
$$

When giving a semantics for subsequence patterns we will use a type indexed function flatten to merge lists of values. It is defined as follows:

$$
\begin{aligned}
& \operatorname{flatten}_{T}(v)=[v] \\
& \text { flatten }_{[\tau]}([])=[] \\
& \text { flatten }_{[\tau]}(v, v s)=\text { flatten }_{\tau}(v)+\text { flatten }_{[\tau]}(v s) \\
& \text { flatten }_{\text {Maybe }}(\text { Nothing })=[] \\
& \text { flatten }_{\text {Maybe } \tau}(\text { Just } v)=\text { flatten }_{\tau}(v) \\
& \text { flatten }_{\text {Either } \tau_{1} \tau_{2}}(\text { Left } v)=\text { flatten }_{\tau_{1}}(v) \\
& \text { flatten }_{\text {Either } \tau_{1} \tau_{2}}(\text { Right } v)=\text { flatten }_{\tau_{2}}(v)
\end{aligned}
$$

We will refer to the set of bound variables in a pattern $p$ as

\subsection{Semantics for linear patterns}

The semantics for linear regular expression patterns can be found in figure 2. Due to space reasons we only give a few of the rules as we explain below.

The judgement for matching linear patterns is denoted $l \in l p \rightarrow$ $v ; \beta ; l$ '. It should read as " $l$ is matched by a pattern $p$ yielding a value $v$, a set of variable bindings $\beta$, and a remainder list $l^{\prime}$ ". $l$ and $l^{\prime}$ range over Haskell lists, where $l$ is the list we wish to match and $l^{\prime}$ is a (possibly empty) suffix of $l$ that wasn't matched.

First of all we have a rule HM-REGPAT that extends Haskell's pattern matching semantics, denoted $\epsilon_{h}$, with regular expression patterns. It does so by performing a linear match.

$$
\frac{l \in_{l}\left(/ p_{1} \ldots p_{n} /\right) \rightarrow l ; \beta ;[]}{l \in_{h}\left[p_{1} \ldots p_{n}\right] \rightarrow \beta}
$$

Here we require that the remainder list is empty i.e. that the whole input list is succesfully matched. This requirement together with the ordering on the rules determines which derivation must be chosen.

The base rule, LM-BASE, is that where the pattern to match is a normal Haskell pattern. In this case we piggy-back on Haskell's normal mechanism for binding variables from patterns.

$$
\frac{e \in_{h} \pi \rightarrow \beta}{e: l \in_{l} \pi \rightarrow e ; \beta ; l}
$$

Apart from ordinary Haskell patterns there are two ways that we can bind variables to values at toplevel, given by the rules LM-AS and LM-ACCAs. The a operator simply binds the variable to a value, whereas the $A$ : operator binds the variable to a list containing the value. The behavior of $a$ : clearly makes more sense in a non-linear context, where the number of bound values may vary, but since it is harmless to do so we have chosen to allow it to appear in linear contexts as well.

For subsequences we simply match each pattern in the sequence in order, as stated by the rule LM-SEQ. The values produced after matching are concatenated and the resulting disjoint sets of variable bindings are merged. The value yielded by matching a subsequence should always be a list of elements, so before we can concatenate the values of the sub-matches we need to flatten these values to simple lists. Here we need to use the typing relation on patterns defined in section 5 . The typing relation is defined relative to some base type $T$ that during the actual matching will be instantiated to the type of the elements in the matching list.

Matching a non-linear pattern in a linear context is identical to matching it in a non-linear context. This is exemplified by the rule 


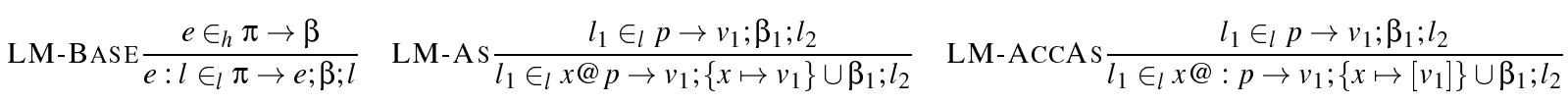

$$
\begin{aligned}
& \text { LM-SEQ } \frac{l_{1} \in_{l} p_{1} \rightarrow v_{1} ; \beta_{1} ; l_{2} \quad \ldots \quad l_{n} \in_{l} p_{n} \rightarrow v_{n} ; \beta_{n} ; l_{f}}{l_{1} \epsilon_{l}\left(/ p_{1} \ldots p_{n} /\right) \rightarrow \gamma_{1}+\ldots+\gamma_{n} ; \beta_{1} \cup \ldots \cup \beta_{n} ; l_{f}} \gamma_{i}=\text { flatten }_{\tau}\left(v_{i}\right), p_{i}: \tau \quad \text { LM-STAR } \frac{l_{1} \in p^{*} \rightarrow v, \beta, l_{2}}{l_{1} \in \in_{l} p^{*} \rightarrow v ; \beta, l_{2}} \\
& \text { HM-REGPAT } \frac{l \in_{l}\left(/ p_{1} \ldots p_{n} /\right) \rightarrow l ; \beta ;[]}{l \in_{h}\left[p_{1} \ldots p_{n}\right] \rightarrow \beta}
\end{aligned}
$$

Figure 2. Semantics for linear regular expression patterns

LM-STAR. The rules for the rest of the operators are similar and are left out due to space restrictions.

\subsection{Semantics for non-linear patterns}

The relation for matching in a non-linear context, denoted $l \in p \rightarrow$ $v ; \beta ; l^{\prime}$ (the only difference in syntax is that we drop the subscript on $\in$ ), is similar to the relation for linear contexts. It differs in two crucial aspects, namely variable bindings and that we handle nonlinear patterns. The rules can be found in figure 3 .

The base rule M-BASE is once again that where the pattern to match is an ordinary Haskell pattern. Since the matching now takes place in a non-linear context, the values of variables being bound while matching this pattern are put into lists instead of just being bound outright. Binding variables explicitly in a non-linear context can only be done using the $\mathrm{e}$ : (accumulating as) operator that binds its variable argument to a list of the value matching its pattern argument, as shown in the rule M-ACCUMAs.

The rule for matching a subsequence, M-SEQ, is identical to LMSEQ except that subpatterns in the sequence are also matched in a non-linear context.

The rules for a repetition pattern, M-STAR1 and M-STAR2, give a non-greedy semantics to the operator by giving the rule for not matching higher precedence than the rule for actually matching the subpattern. The first rule simply doesn't try to match anything, whereas the second rule matches the given subpattern $p$ once and then recurses to obtain more matches. The value obtained from matching $p$ is then prepended to the result values of the recursive second premise. Similarly the values of bound values are prepended to the bindings from the recursive call. To get a greedy semantics in the rules M-GSTAR 1 and M-GSTAR 2 we simply swap the order of the rules to give precedence to preforming a match.

The non-empty repetition pattern operator $p^{+}$is defined as $p^{+} \equiv$ $p p^{*}$, similarly its greedy counterpart $p^{+} ! \equiv p p^{*} !$, and the rules MPLUS and M-GPLUS can easily be derived from these facts.

The rules M-OPT 1 and M-OPT2 for optional patterns are very similar to the rules for repeating patterns, only that no recursion to obtain more matches is done. The values returned by an optional pattern are of the Haskell Maybe type for optional values.

For choice regular expression patterns we return values of the Haskell Either type to indicate which choice was taken. In the rules M-CHOICE1 and M-CHOICE2 we give precedence for matching the left pattern. Furthermore all variables occuring only in the branch not taken are assigned empty lists.

\section{Well-formed regular expression patterns}

We now turn our attention to the static semantics of regular expression patterns. We will refer to the static semantics as wellformedness of regular expression patterns.

There are two reasons why we need a static semantics. The first reason concerns where and how a variable is bound in a pattern. In ordinary patterns a variable may appear only once, with the notable exception for or-patterns found in Ocaml and SML/NJ. In these languages all alternatives must bind exactly the same set of variables. We have similar yet more liberal restrictions on variable bindings. Bound variables must not necessarily be bound in all alternatives in a choice pattern.

The second reason is that we need to ensure that the types of the bound variables are correct. The same variable should in particular have the same type for all its occurrences in a choice pattern.

To express the well-formedness of a regular expression pattern we use the judgment $\Delta \vdash_{l} p$ which says that a (linear) regular expression pattern $p$ is well-formed in the typing context $\Delta$. The typing context $\Delta$ gives types to the variables bound in the pattern. When checking the validity of patterns in a non-linear context we use the judgment $\Delta \vdash p$ which is similar to the judgment for linear patterns. We will also refer to the well-formedness of patterns in Haskell, using the judgment $\Delta \vdash_{h} p$. We refer to Faxén's paper for a static semantics of Haskell patterns [Fax02]. We require that $\Delta \vdash_{h} p$ can only be derived if $p$ binds exactly the variables in the typing context $\Delta$. Finally we will need a notion of types for regular expression patterns. We use the judgment $p:: \tau$ to say that the pattern $p$ has the type $\tau$.

Checking the well-formedness of a regular expression pattern as an ordinary pattern in the host language is done using the following rule. Is is noteworthy that we split the typing context. All the typing contexts $\Delta_{i}$ must bind different names. We use this to enforce that a variable may only be bound once.

$$
\frac{\Delta_{1} \vdash_{l} p_{1} \ldots \Delta_{n} \vdash_{l} p_{n}}{\Delta_{1} \ldots \Delta_{n} \vdash_{h}\left[p_{1} \ldots p_{n}\right]} \Delta_{i} \cap \Delta_{j}=\emptyset \forall i j . i \neq j
$$

The rules for establishing well-formedness of linear patterns can be found in figure 4 . In this section we only present the rules for nongreedy operators as the rules for greedy counterparts are exactly the same. The only interesting thing to note about the rules for $*,+$ and ? is the fact that when checking their subpatterns we are in a nonlinear context and therefore use the corresponding judgment for the premises. The rule for sequences is reminiscent of that for regular expression patterns in the context of ordinary patterns explained above. 


$$
\begin{aligned}
& \operatorname{M-BASE} \frac{e \in_{h} \pi \rightarrow \beta}{e: l \in \pi \rightarrow e ; \sigma ; l} \sigma=\{x \mapsto[v] \mid x \mapsto v \in \beta\} \quad \text { M-ACCAs } \frac{l_{1} \in p \rightarrow v_{1} ; \beta_{1} ; l_{2}}{l_{1} \in x @: p \rightarrow v_{1} ;\left\{x \mapsto\left[v_{1}\right]\right\} \cup \beta_{1} ; l_{2}} \\
& \operatorname{M-SEQ} \frac{l_{1} \in p_{1} \rightarrow v_{1} ; \beta_{1} ; l_{2} \quad \ldots \quad l_{n} \in p_{n} \rightarrow v_{n} ; \beta_{n} ; l_{f}}{l_{1} \in\left(/ p_{1} \ldots p_{n} /\right) \rightarrow \gamma_{1}+\ldots+\gamma_{n} ; \beta_{1} \cup \cdots \cup \beta_{n} ; l_{f}} \gamma_{i}=\operatorname{flatten}_{\tau}\left(v_{i}\right), v_{i}:: \tau \\
& \operatorname{M-STAR} 1 \frac{p^{*} \rightarrow[] ; \beta ; l}{l \in} \beta=\{x \mapsto[] \mid x \in \operatorname{vars}(p)\} \quad \operatorname{M-STAR} 2 \frac{l_{1} \in p \rightarrow v_{1} ; \beta_{1} ; l_{2} \quad l_{2} \in p^{*} \rightarrow v_{2} ; \beta_{2} ; l_{3}}{l_{1} \in p^{*} \rightarrow v_{1}: v_{2} ; \beta_{1} \uplus \beta_{2} ; l_{3}} \\
& \operatorname{M-GStAR} 1 \frac{l_{1} \in p \rightarrow v_{1} ; \beta_{1} ; l_{2} \quad l_{2} \in p^{*} ! \rightarrow v_{2} ; \beta_{2} ; l_{3}}{l_{1} \in p^{*} ! \rightarrow v_{1}: v_{2} ; \beta_{1} \uplus \beta_{2} ; l_{3}} \quad \operatorname{M-GSTAR} 2 \frac{}{l \in p^{*} ! \rightarrow[] ; \beta ; l} \beta=\{x \mapsto[] \mid x \in \operatorname{vars}(p)\} \\
& \text { M-PLus } \frac{l_{1} \in p \rightarrow v_{1}, \beta_{1}, l_{2} \quad l_{2} \in p^{*} \rightarrow v_{2}, \beta_{2}, l_{3}}{l_{1} \in p^{+} \rightarrow v_{1}: v_{2}, \beta_{1} \uplus \beta_{2}, l_{3}} \quad \text { M-GPLUS } \frac{l_{1} \in p \rightarrow v_{1}, \beta_{1}, l_{2} \quad l_{2} \in p^{*} ! \rightarrow v_{2} ; \beta_{2} ; l_{3}}{l_{1} \in p^{+} ! \rightarrow v_{1}: v_{2}, \beta_{1} \uplus \beta_{2}, l_{3}} \\
& \text { M-OPT } 1 \frac{l_{1} \in p \rightarrow v_{1}, \beta_{1}, l_{2}}{l \in p ? \rightarrow \text { Nothing, } \beta, l} \beta=\{x \mapsto[] \mid x \in \operatorname{vars}(p)\} \quad \text { M-Opt } 2 \frac{l_{1}}{l_{1} \in p ? \rightarrow\left(\text { Just } v_{1}\right), \beta_{1}, l_{2}} \\
& \operatorname{M-GOPT} 1 \frac{l_{1} \in p \rightarrow v_{1}, \beta_{1}, l_{2}}{l_{1} \in p ? ! \rightarrow\left(\text { Just } v_{1}\right), \beta_{1}, l_{2}} \quad \text { M-GOPT } 2 \frac{}{l \in p ? ! \rightarrow \text { Nothing, } \beta, l} \beta=\{x \mapsto[] \mid x \in \operatorname{vars}(p)\} \\
& \operatorname{M-Choice~} 1 \frac{l_{1} \in p_{1} \rightarrow v_{1} ; \beta ; l_{2}}{l_{1} \in\left(p_{1} \mid p_{2}\right) \rightarrow\left(\text { Left } v_{1}\right) ; \sigma ; l_{2}} \sigma=\beta \cup\left\{x \mapsto\left[\mid x \in \operatorname{vars}\left(p_{2}\right)\right\} \backslash \operatorname{vars}\left(p_{1}\right)\right) \\
& \text { M-Choice } 2 \frac{l_{1} \in p_{2} \rightarrow v_{1} ; \beta_{1} ; l_{2}}{l_{1} \in\left(p_{1} \mid p_{2}\right) \rightarrow\left(\text { Right } v_{1}\right) ; \beta ; l_{2}} \beta=\beta_{1} \cup\left\{x \mapsto\left[\mid x \in \operatorname{vars}\left(p_{1}\right)\right\} \backslash \operatorname{vars}\left(p_{2}\right)\right)
\end{aligned}
$$

Figure 3. Semantics for non-linear regular expression patterns

$$
\begin{gathered}
\frac{\Delta \vdash p}{\Delta \vdash_{l} p^{*}} \quad \frac{\Delta \vdash p}{\Delta \vdash_{l} p^{+}} \quad \frac{\Delta_{1} \vdash p \Delta_{2} \vdash q}{\Delta \vdash_{l} p \mid q} \Delta=\Delta_{1} \cup \Delta_{2} \quad \frac{\Delta \vdash p}{\Delta \vdash_{l} p ?} \\
\frac{\Delta_{1} \vdash p_{1} \ldots \Delta_{n} \vdash p_{n}}{\Delta_{1} \ldots \Delta_{n} \vdash_{l}\left(/ p_{1} \ldots p_{n} /\right)} \Delta_{i} \cap \Delta_{j}=\emptyset \forall i j . i \neq j \\
\frac{p:: \tau \quad \Delta \vdash_{l} p}{\Delta, x:: \tau \vdash_{l} x @ p} \quad \frac{p:: \tau \quad \Delta \vdash_{l} p}{\Delta, x::[\tau] \vdash_{l} x @: p} \quad \frac{\Delta \vdash_{h} h p a t}{\Delta \vdash_{l} h p a t}
\end{gathered}
$$

Figure 4. Wellformed linear regular expression patterns

The variable binding rules are interesting to contrast against each others. "As"-patterns are well-formed if the variable is bound to a pattern with the same type as the variable. "Accumulating as"patterns on the other hand may match several times so the type of the variable must be a list.

In figure 5 we present the rules for establishing the well-formedness of non-linear patterns. Most of the rules carry over straightforwardly from those for linear patterns. It should be noted though that the rule for ordinary patterns rebuilds the typing context so that all variables have list types.

Figure 6 gives the typing rules for regular expression patterns. The intuition behind these rules is that a pattern has a type which reflects the ways it can match. For example a pattern which can match many times has a list type, hence variables bound to $*$ and + patterns get list types. Choice patterns can match one of two things

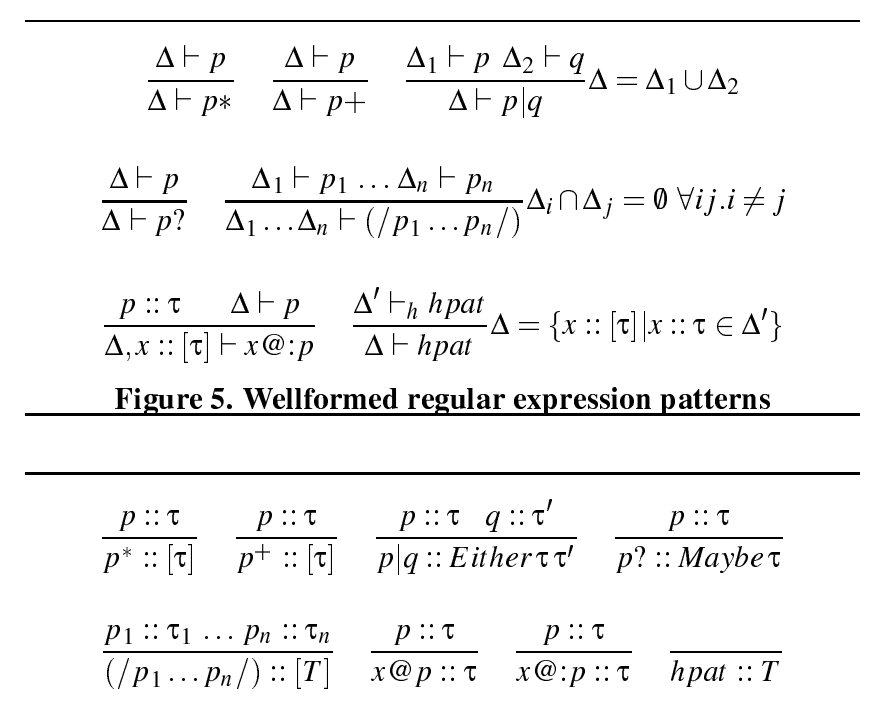

Figure 6. Typing rules for regular expression patterns

which is captured by the Either type of Haskell. A sequence pattern matches yields a sequence and hence it also has a list type. Variable binding patterns don't affect the typing. The last typing rule for ordinary patterns in the underlying language is more surprising, since it refers to a specific type $\mathrm{T}$. This means that the typing rules should be interpreted in a context where we are matching on a list of type $[\mathrm{T}]$, i.e. $\mathrm{T}$ is the type of the elements of the list. 


\section{Implementation}

We currently have an implementation of our regular expression pattern system that works as a preprocessor for GHC. It takes a source code file possibly containing regular expression patterns and translates it into semantically equivalent vanilla Haskell code. It also comes with a matching engine, which we implement as a simple parser monad. The preprocessor does not check any types, instead we rely on GHC's type checker to catch type errors.

\subsection{Matching engine}

The datatype for a matching parser, which we from now on will refer to as a matcher, looks like

data Matcher e a $=\operatorname{Matcher}([e] \rightarrow[(a,[e])])$

It is essentially a function that takes an input list, conducts a match, and returns a list of results. Each result will consist of a value, a set of values for bound variables, and a remainder list. All of this is read directly from our semantic rules.

Since different variables will be bound to values of different types, we need to model the set of bindings as a tuple, with each entry corresponding to the value(s) for one specific variable. As is customary, we let the remainder list be the state of the matcher monad, so that it is implicitly threaded through a series of matches. The individual matcher functions then need to return a value for future bindings, and a tuple with values for variables.

To account for our all-match semantics the parser generates a list of results at each step. At places where we need to branch we can use the +++ operator which lets us proceed with two different matchers. We define +++ as

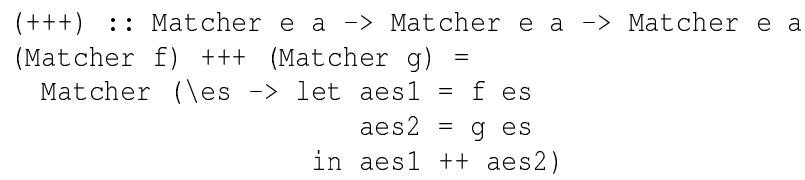

As we can see from the definition +++ is left-biased, i.e. any results from its left operand will end up before any results from its right operand in the list of results. This allows us to define a function that conducts the full matching by, as defined by our first-match policy, selecting the first result in this list of results for which the matcher has reached the end of the input list (i.e. the remainder list is empty). This function, called runMatch, corresponds to the rule HM-REGPAT from figure 2, and is defined as

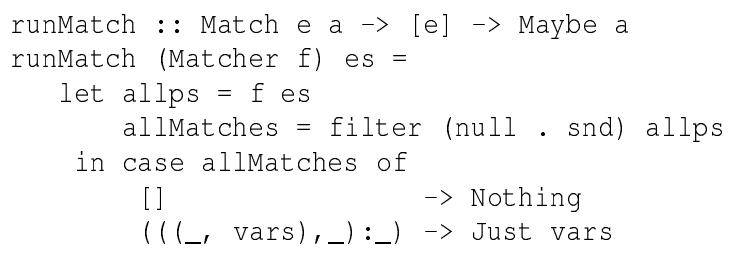

\subsection{Translation}

The basic idea behind translating a regular expression pattern into vanilla Haskell is to generate a matcher for each subpattern, all the way down to ordinary Haskell patterns, and then combine these to form a top-level matcher corresponding to the whole of the pattern.

\subsubsection{Base patterns}

The base case is when the pattern in question is an ordinary Haskell pattern. First we must generate a function that actually takes an element from the input list and tries to match it to the given pattern. For example, if the pattern in question is $\mathrm{Tel} \mathrm{nr}$, the corresponding function would look like

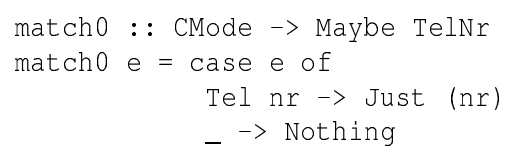

No type signatures are actually generated, we just supply them here to simplify understanding. To avoid overly long signatures we abbreviate ContactMode with CMode in our examples.

What the function returns if the match succeeds is a tuple containing the values of bound variables. The function above works in linear context since we return the bound variable as is. If we instead wanted a function to work in non-linear context, we would wrap the values in lists, like

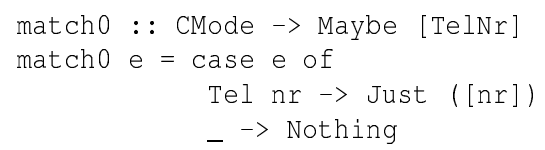

We also need to lift a generated matching function into the matcher monad. This lifting works identically regardless of what the pattern is, so we have a function in the matcher engine that does this, defined as

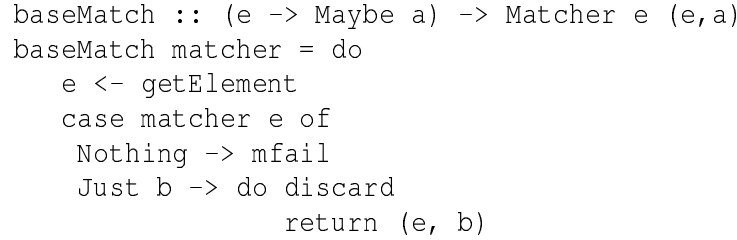

The functions used by baseMatch are inherent to our matcher monad. getelement retreives the head of the input list, discard drops the head of the input list, and mfail is a matcher that always returns an empty list of results. We now need to generate a matcher by applying baseMatch to our generated function, i.e.

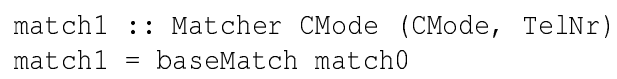

The type states that match 1 is a matcher for a list of CModes. The value matched is a CMode, and the only variable bound is of type TelNr. The numbers 0 and 1 in the names of these functions signify that each name is fresh, i.e. these numbers could be any positive integers, but no two functions share the same integer.

For Haskell patterns that are guaranteed to always match, i.e. pattern variables and wildcards (-), we can simplify these steps. For a wildcard, what we need to generate is the matcher

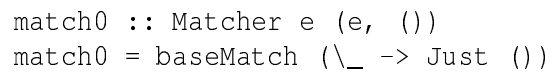

meaning we will always match, and no variables are bound. The only difference for a pattern variable is that the variable in question is also bound, e.g. for the pattern a we get 
match0 : : Matcher e $(e, e)$

matcho $=$ baseMatch $(\backslash a->$ Just (a) $)$

Once again the shown function works in linear context, in nonlinear context we would wrap the returned a in a list.

\subsubsection{Repetition}

All regular expression patterns have one or more subpatterns, and the first step when translating a regular expression pattern will be to translate these subpatterns. For a repetition pattern, $p *$, we would first translate the subpattern $p$ into some matcher function matchX. According to the rules M-STAR 1 and M-STAR2, a matcher for a repetition pattern should if possible continue without trying to match anything, otherwise it should match one element and then recursively match the repetition pattern again. This behavior is common to all repetition patterns so we define it as a function in the matching engine:

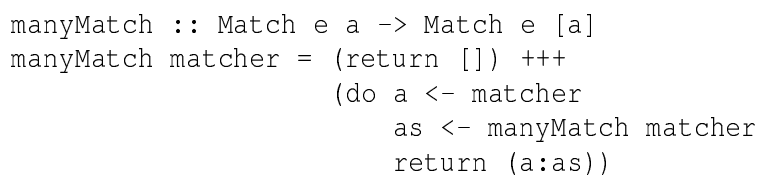

The problem with this definition is that manyMatch returns a list in which each element is the result of one step of the recursion. We need to unpack this list so that we instead return a tuple, in which each entry is a list of results for a specific variable binding. We cannot do this generically since the number of bound variables, and thus the size of the tuple, will vary. Therefore we must supply an appropriate unzipping function that works for the correct number of variables. The exact function to use can be determined by the preprocessor, that has the necessary meta-information on what variables are bound. Note that all variables inside the repetition will be non-linear, so the result of matching a variable in each step of the recursion will be a list of values. If we only unzip to get a list of such results for each variable, what we would really get is a list of lists of values. Thus to get a list of values we should also let the unzipping function concatenate the results for each variable in the resulting tuple.

Inside manyMatch the unpacking will be done in two steps. The first is to simply unzip the list into two lists, one containing all values $\left(v_{i}\right.$ from the rules), the other containing all values of bound variables. In the second step we need to apply the supplied unzipping-andconcatenating function to the latter list to get the variable values proper. This new improved manyMatch will thus look like

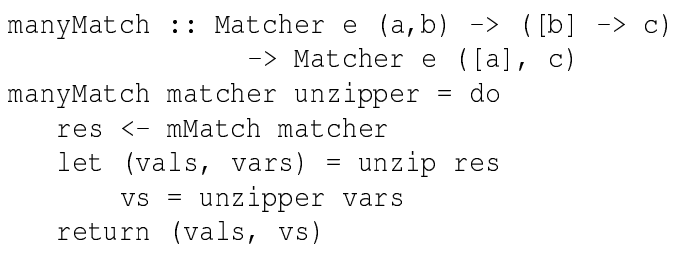

where mMatch is our old definition of manyMatch.

As an example, we show the translation of the pattern (Tel nr)*. The first step is to translate the subpattern $\mathrm{Tel}$ a, which we have already seen how to do. The new function that we generate will then look like

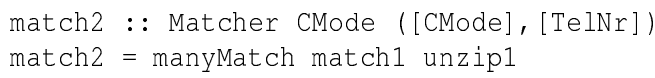

assuming the matcher for the subpattern is called match1. The function unzip1 here is simply the concat function, since there is only one variable bound. To account for the greedy version of a repetition pattern, *!, we simply flip the arguments to +++ in manyMatch, which will give a higher priority to the case when we actually match an element.

Non-empty repetition patterns, + , are very similar to ordinary repetition patterns, the only difference is of course that we make an initial match before starting the recursion, as shown in

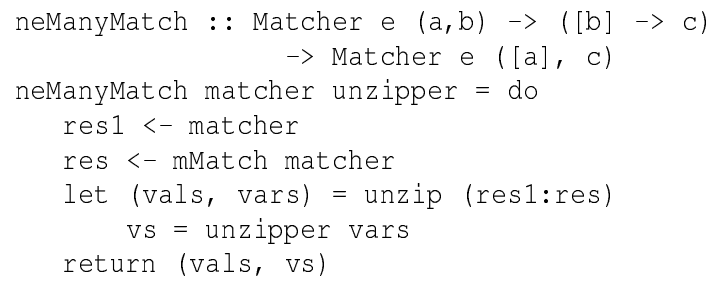

\subsubsection{Choice and Optional patterns}

Choice patterns are slightly trickier to handle because of the way variables are bound. As we saw in the rules M-CHOICE 1 and MCHOICE2, any variables appearing in the other branch than the one being matched should be bound to empty lists. This is very difficult to handle generically since we need access to the meta-information of variable names. Thus we instead generate the full code for the choice pattern during translation. As an example we translate the pattern ( $\mathrm{Tel} \mathrm{nr}$ | Email eaddr). We start by translating the subpatterns, resulting in two functions that we assume are named match 1 and match2. The code generated for the choice pattern will be

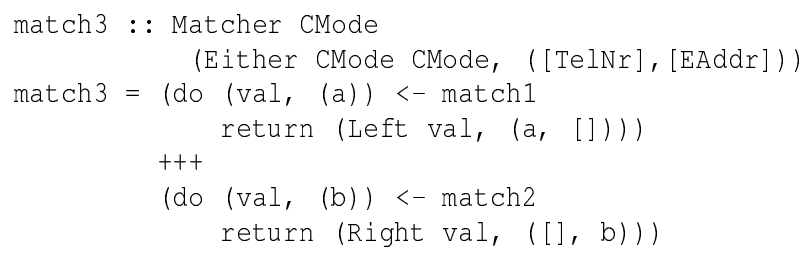

where we have tagged the result value of the pattern match with the respective constructors from the Either type.

The story is very similar for optional patterns, but this time all variables should be bound to empty lists if no match is done. For the pattern ( $\mathrm{Tel} n \mathrm{n}$ ) ? we get

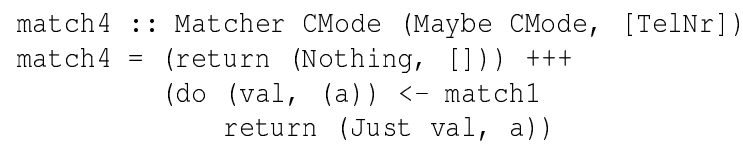

For a greedy optional pattern we would simply switch the arguments to +++ , just as for repetition patterns.

\subsubsection{Subsequences}

The trickiest pattern to implement is subsequence, due to the need for flattening. As we saw in section 5, flattening is done based on the type of a subpattern (with respect to some base type for elements in the input list), which means that the preprocessor must keep track of these types in order to insert the proper flattening functions. For a pattern (/ (Tel nr)?, (Email eaddr)* /) we get 
the following translation, assuming the two subpatterns are translated into matcher functions match1 and match2 respectively:

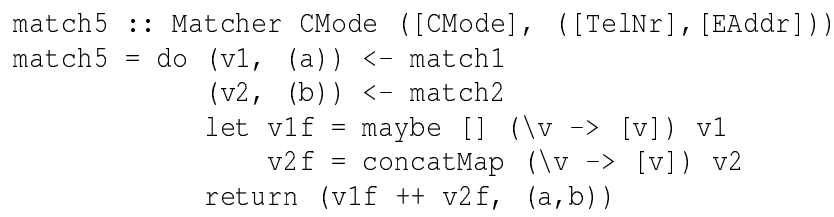

The value $\mathrm{v} 1$ is the result of match1, i.e. the matcher for ( $\mathrm{Tel}$ nr) ?, so it will have type Maybe CMode. To flatten it we use the built-in Haskell function maybe that takes two arguments, one that is a default value to return if it encounters a Nothing (in this case [ ] ), the other a function to apply to a value held by a Just (in this case the flattening function for a value of the base type). Similarly v2 comes from match2, so its type will be [CMode]. We flatten it using the built-in function concatMap that takes a function, applies is to all elements of a list, and then concatenates the results.

\subsubsection{Variable bindings}

Finally we turn to the explicit binding operators. Binding a variable to a value in our matcher means to add that value to the result tuple. Since an explicitly bound variable syntactically appears to the left of any variables in its subpattern, we add the value in the leftmost position in the tuple, i.e. before those bound in the subpattern. Thus we know that the values in the result of the top-level matcher should be bound to variables from left to right in the order they appear in the pattern. As an example consider the pattern ae $(\mathrm{Tel}$ nr | Email eaddr). We first translate the subpattern (Tel nr | Email eaddr) into a matcher match1. The matcher generated for the variable binding will then be

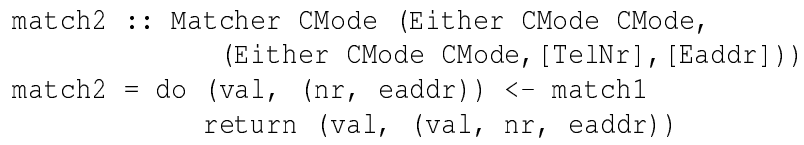

If we had instead used non-linear binding, i.e. al: (Tel $\mathrm{nr}$ | Email eaddr), we would get a list for the returned value, i.e.

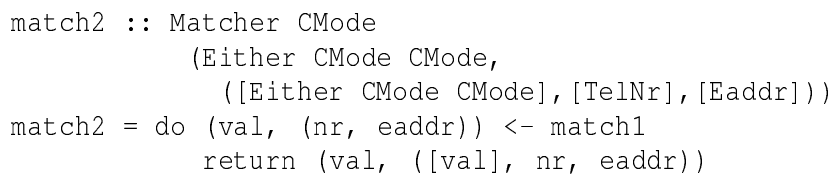

\subsection{Matching}

Now we know how to translate a regular expression pattern into a top-level matcher function, what is left is to insert and invoke the generated matcher at the right place to preserve the pattern matching semantics. To this end we use Haskell pattern guards [EPJ00] that allow us to evaluate a function and pattern match on the result as part of the original pattern match. The function that we so wish to evaluate is runMatch applied to our generated top-level matcher and the input list that we wish to match. For our matcher functions to be in scope we add them to the where clause of the declaration that the regular expression pattern appears in. To show a complete example of the translation of a function declaration we revisit our function allTels defined as

$$
\text { allTels (Person _ }\left[\left(\operatorname{Tel} n r \mid{ }^{*}\right]\right)=n r
$$

since it contains several different features of regular expression patterns. The translated version of this function will look like

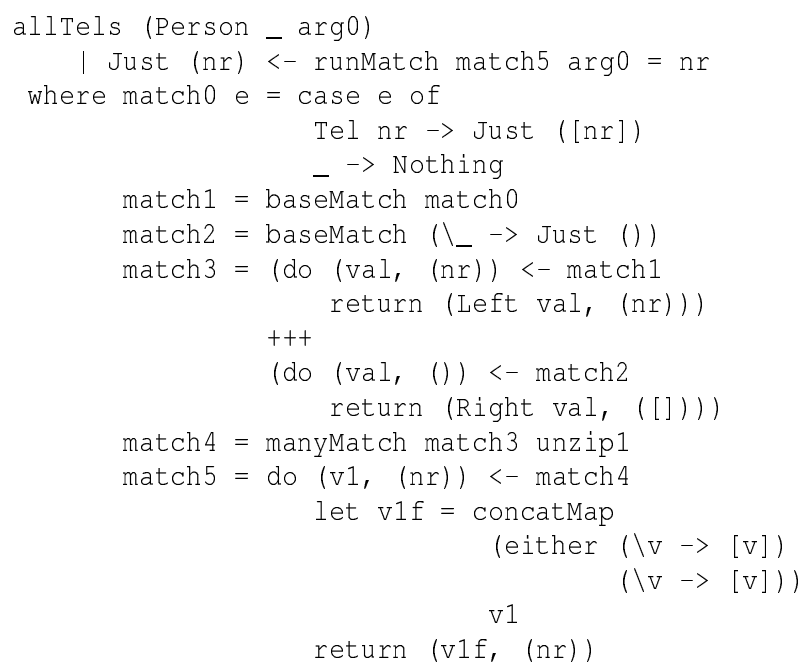

The functions match 0 and match1 together correspond to the pattern $(\mathrm{Tel} n \mathrm{n})$. Note the list around the returned variable nr signaling that the pattern is matched in a non-linear context. match2 corresponds to the pattern _. Combining these two into a choice patterns yields ( $\mathrm{Tel} n r \mid{ }_{-}$), which is translated to match3. On top of that we add a repetition, which gives us match 4 when translated. Finally since the top-level pattern should be matched as a subsequence, as seen in the rule HM-REGPAT, we translate it into match5. The actual matching is done in the pattern guard that applies runMatch to the matcher and the input list. The latter is held by an automatically generated fresh variable, in this case arg0. It is also interesting to note that the actual binding of variables to values does not happen until runMatch is evaluated. Any mention of variable names in the matcher functions, e.g. nr in matcho, are only there as mnemonic aids to a human reader. We could change all such names to freshly generated variable names without changing any semantics.

In Haskell, patterns can appear in numerous places such as function declarations, case expressions, let expressions, statements etc. Translating regular expression patterns into vanilla Haskell is slightly different depending on just where the pattern appears. The generated matchers will be identical in all cases, but the placement of them and of the evaluation may differ. We will not go through these differences in detail, but our implementation handles all cases correctly. Irrefutable (lazy) patterns also require special care, and we have yet to implement support for them in full.

\section{Related Work}

Pattern matching is a well-known and much studied feature of functional languages [Aug85, Wad87, Mar92, Mar94]. It provides the startingpoint for the work presented in this paper.

Regular expressions have been used in programming for a long time, mostly for text matching purposes. Perl's support for regular expressions is probably one of the most well-known [Perl], but most mainstream languages, including Haskell, have some library support for regular expression text matching. Regular expressions in such libraries are themselves encoded as strings. Matching them means taking two strings, where one encodes a regular expression, and match them to each other. This is in some sense very low- 
level when compared to our regular expression patterns since there are no guarantees that regular expressions encoded as strings are well-formed, and there is no direct way to bind variables to values during a match. Yet another drawback is of course that such regular expressions work on strings only, whereas our regular expression patterns work over lists of any datatype.

The recent trend in XML-centric languages has led to several new languages with support for regular expression pattern matching such as XMLambda [MS99], XDuce [HP03] and CDuce [BCF03]. Most similar to ours is probably CDuce, a general purpose XMLcentric programming language. The main focus in this language is its regular expression types which are used to validate XML documents. Borrowing from XDuce they also have regular expression patterns which are tightly coupled with the type system. This allows for very precise type information to be propagated in the right hand side of a pattern. The main difference with our work is the close connection with the type system. Our extension is little more than just syntactic sugar which makes it very easy to implement.

Another recently developed language that features regular expression patterns is Scala [Scala]. Scala is a multi paradigm language supporting both object oriented and functional programming. Its regular expression facility is rather similar to ours but differs at the following points. Firstly, there is only one variable binding construct which has a context dependent behaviour. Secondly, Scala has non-greedy operators just as we do but have no greedy counterparts. This can make some patterns awkward to express. Scala's regular expression patterns work for arbitrary sequences.

There has been some work in extending Haskell with the full power of XDuce, called XHaskell [LS04]. This work focuses on fitting the type system of XDuce into Haskell and encoding it using Haskell's class system. They also have regular expression patterns but these are intimately coupled with regular expression types and do not work together with ordinary pattern matching.

\section{Future Work}

There are several areas where our regular expression patterns extension can be improved. It is not obvious that our implementation using a monadic parser is the most efficient approach, on the contrary. There has been lots of work on efficient matching of regular expressions and it is likely that some of these techniques could be used with our system to make it more efficient.

We will need to devise and implement a type checking algorithm for our regular expression patterns on top of Haskell's type checking mechanism. Being able to type check our regular expression patterns before translating them into vanilla Haskell, as opposed to our current implementation that first translates and then lets a Haskell type checker do the work, would, if nothing else, lead to much improved error messages.

\section{Acknowledgement}

We would like to thank our shepherd Erik Meijer for his many suggestions which improved the paper enormously. Thanks also to Karol Ostrovsky and David Sands who gave valueable feedback on draft versions of this paper. The participants of the Multi Meeting provided insightful comments when we presented the material in this paper. Lastly thanks to the anonymous referees for their comments.
This work was partially funded by the Swedish Foundation for Strategic Research.

\section{References}

[Aug85] Lennart Augustsson. Compiling Pattern Matching. In Functional Programming and Computer Architecture, 1985.

[BCF03] V. Benzaken, G. Castagna, and A. Frisch. CDuce: An XML-Centric General-Purpose Language. In Proceedings of the ACM International Conference on Functional Programming, 2003.

[EPJ00] Martin Erwig and Simon Peyton Jones. Pattern Guards and Transformational Patterns. In Haskell Workshop, 2000.

[Fax02] Karl-Filip Faxén. A Static Semantics for Haskell. Journal of Functional Programming, 12(4-5), 2002.

[Fri04] A. Frisch. Regular Tree Language Recognition with Static Information. In 3rd IFIP International Conference on Theoretical Computer Science, 2004.

[HM03] Haruo Hosoya and Makoto Murata. Boolean Operations and Inclusion Test for Attribute-Element Constraints. In Eighth International Conference on Implementation and Application of Automata, volume 2759 of Lecture Notes in Computer Science, pages 201-212. Springer-Verlag, 2003.

[HP03] Haruo Hosoya and Benjamin C. Pierce. XDuce: A Typed XML Processing Language. ACM Transactions on Internet Technology, 2(3):117-148, 2003.

[HVP00] Haruo Hosoya, Jerome Vouillon, and Benjamin C. Pierce. Regular Expression Types for XML. In Proceedings of the ACM International Conference on Functional Programming, 2000.

[Lev03] Michael Y. Levin. Compiling Regular Patterns. In Proceedings of the ACM International Conference on Functional Programming, pages 65-78, 2003.

[LS04] Kenny Zhuo Ming Lu and Martin Sulzmann. XHaskell: Regular Expression Types for Haskell. http://www.comp.nus.edu.sg/ sulzmann/, 2004.

[Mar92] Luc Maranget. Compiling Lazy Pattern Matching. In Proc. of the 1992 conference on Lisp and Functional Programming. ACM Press, 1992.

[Mar94] Luc Maranget. Two Techniques for Compiling Lazy Pattern Matching. Research report 2385, INRIA, 1994.

[MS99] Erik Meijer and Mark Shields. XM $\lambda$ : A Functional Language for Constructing and Manipulating XML Documents. (Draft), 1999.

[MvV01] Erik Meijer and Danny van Velzen. Haskell Server Pages. In Graham Hutton, editor, Electronic Notes in Theoretical Computer Science, volume 41. Elsevier, 2001.

[Perl] www.perl.org.

[Scala] Martin Odersky et.al. The Scala Programming Language. http://scala.epfl.ch/.

[Wad87] Philip Wadler. The Implementation of Functional Programming Languages, chapter Efficient Compilation of Pattern Matching. Prentice Hall, 1987. 